\title{
Over $10 \%$ efficiencies achieved for the PSCs with thick active layer based on D-A copolymer donors and various fullerene acceptors
}

In comparison with inorganic solar cells, polymer solar cells (PSCs) possess the advantages of light weight and potential low-cost production technology such as roll-to-roll printing production. And the power conversion efficiency (PCE) of the PSCs reached ca. 10\% recently. However, the high PCE is limited to the PSCs with the low bandgap polymer PBDTTTs as donor and PC70BM as acceptor, and the optimized active layer thickness of the high efficiency PSCs is usually less than $100 \mathrm{~nm}$. The dominant acceptor of PC70BM places serious limitation on the development of the polymer donor materials to match with PC70BM, and the thin active layer makes large area fabrication of the PSCs very difficult.

Recently, Prof. He Yan's group at Hong Kong University of Science and Technology, in collaboration with Prof. Harald Ade and Dr. Wei Ma from North Carolina State University, broke the limitation [1]. They synthesized three D-A copolymers with quaterthiophene as donor unit, PffBT4T2OD, PBTff4T-2OD and PNT4T-2OD (Figure 1). The polymers show high crystallinity and high hole mobility of $1.5 \times 10^{-2}-3.0 \times 10^{-2} \mathrm{~cm}^{2} /(\mathrm{Vs})$. The PCE of all the PSCs based on the polymers as donor and 6 fullerene derivatives of ICMA, TC71BM, TC61PM, PC71BM, PC61BM or PC61PM as acceptor reached around $10 \%$, and the active layer of the high efficiency PSCs can be as thick as $300 \mathrm{~nm}$ which is very important for future large area fabrication of the PSCs. The highest PCE reached $10.8 \%$ for the PSC based on PffBT4T-2OD/TC71BM, which is a new record for single- junction PSCs reported to date. The highest fill factor of the devices reached 0.77 for the PSC based on PffBT4T-2OD/ ICMA with a PCE of $9.8 \%$ and a slightly higher open- circuit voltage of $0.78 \mathrm{~V}$ which should be benefited from the higher LUMO of ICMA [2]. The high PSC performances are achieved via the formation of an 'optimum PSC morphology' that contains highly crystalline, sufficiently pure and reasonably small polymer domains. Furthermore, the near ideal morphology is controlled by the temperature- dependent aggregation behavior of the donor polymers and is rather insensitive to the presence of fullerene derivatives. The broad selectivity of the fullerene derivative acceptors should be benefited from the crystallinity
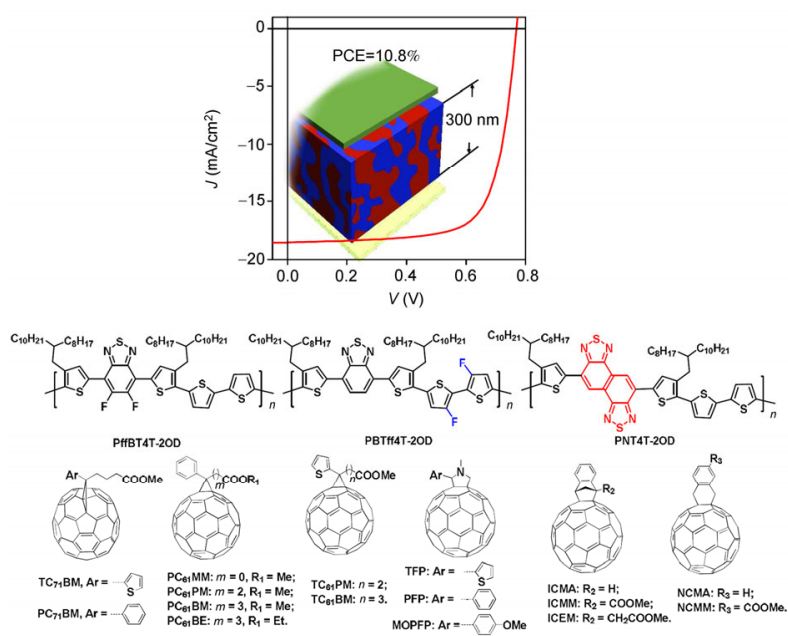

Figure 1 Photovoltaic performance and molecular structures of the polymers donor and fullerene derivatives acceptor.

and aggregation behavior of the polymers.

The key molecular design motif that enables the highly temperature dependent aggregation property of the polymers is the 2nd position branched alkyl chains (2OD, 2octyldodecyl) on a quaterthiophene (4T-2OD). With the revealed aggregation and morphology control approach and polymer design rationales, the PSC community will be able to explore more polymers and fullerene materials and to optimize their combinations under a well-controlled morphological landscape, which could greatly accelerate the development of new photovoltaic materials and device processing technologies towards high performance PSCs.

Yongfang Li

Institute of Chemistry, Chinese Academy of Sciences

1 Liu Y, Zhao J, Li Z, Mu C, Ma W, Hu H, Jiang K, Lin H, Ade H, Yan H. Aggregation and morphology control enables multiple cases of high-efficiency polymer solar cells. Nat Commun, 2014, 5: 5293

2 He Y, Chen H, Hou J, Li Y. Indene-C60 bisadduct: a new acceptor for high performance polymer solar cells. J Am Chem Soc, 2010, 132: 1377-1382 